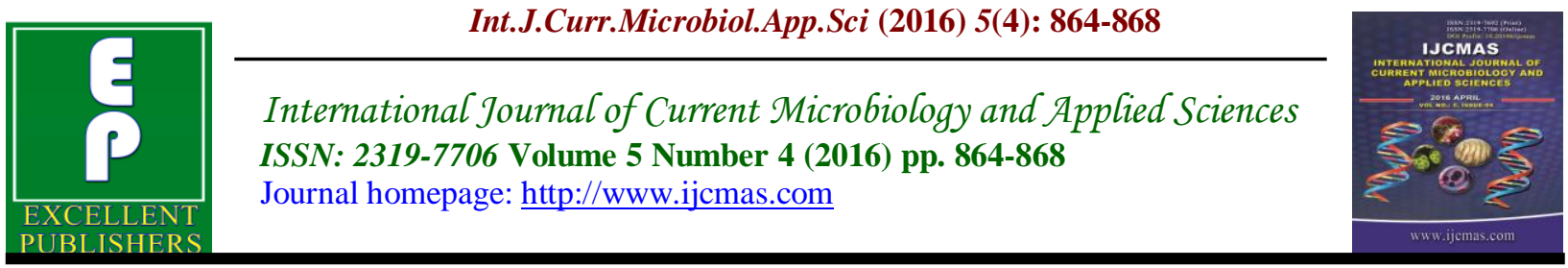

Original Research Article

http://dx.doi.org/10.20546/ijcmas.2016.504.098

\title{
Prevalence of Hepatitis B surface antigen among blood donors and hospital camp attendees at a tertiary care hospital, Hyderabad, India
}

\author{
Md Wajid* and Md Khaleel \\ Department of Microbiology, Deccan College of Medical Sciences, Hyderabad, \\ Telangana State, India \\ *Corresponding author
}

Keywords

Blood donors, Hepatitis B surface antigen, Hospital camp attendees, Prevalence, Seropositivity

\section{Article Info}

Accepted:

20 March 2016 Available Online: 10 April 2016

\section{A B S T R A C T}

Viral Hepatitis is a major public health problem in India. Surveys for screening HBs Ag have been primary, simple and the most useful mode of determining HBV infection rates. The study has been designed with an objective to assess the prevalence of HBs Ag in blood donors and hospital camp attendees. The study was conducted in Owaisi Hospitals which is a tertiary care super speciality hospital. The study population included all the blood donors donating blood in the blood bank of the hospital and hospital camp attendees in the study period of 6 months from February 2015 to July 2015. Standard procedures were used during the blood collection. A total of 2,500 donor blood samples \& 500 hospital camp attendees were screened by Central Drugs Standard Control Organization (CDSCO) approved enzyme linked immuno-sorbent assay (ELISA) kit Hepalisa. Seropositivity was more among hospital camp attendees (4.4\%) compared to blood donors $(1.2 \%)$ which was found to be statistically significant $(\mathrm{p}<0.0001)$. Majority (93.3\%) were males and half the proportion (53\%) belonged to 31-35 years age group. In comparison with voluntary donors, seropositivity was more among replacement donors. A higher rate of prevalence of $\mathrm{HBs} \mathrm{Ag}$ positivity was observed among hospital camp attendees compared to blood donors $4.4 \%$ and $1.2 \%$ respectively showing a higher prevalence in hospital camp attendees.

\section{Introduction}

Viral Hepatitis is a major public health problem in India. ${ }^{1}$ Viral hepatitis can be acute, sub-acute or chronic and the viruses responsible are Hepatitis A (HAV),Hepatitis $\mathrm{B}(\mathrm{HBV})$, Hepatitis C (HCV), Hepatitis D (HDV) and Hepatitis E (HEV).

Hepatitis B virus infection (HBV) is global public health problem. Nearly Two billion people globally have been infected with
HBV,350 to 400 million people chronically infected with HBV and tens of millions of new cases occur annually. ${ }^{2-4}$ At least 15$20 \%$ of chronically HBV infected will die due to liver disease caused by HBV and this constitutes nearly one million people each year. It is the most common cause for chronic liver disease including cirrhosis of the liver and hepatocellular carcinoma worldwide. 
Prevalence of HBV infection varies greatly in different parts of the world. The World Health Organization (WHO) has classified HBV prevalence into high endemicity $(>8 \%)$, intermediate $(2-7 \%)$ and low endemicity $(<2 \%) .^{5} \mathrm{HBV}$ prevalence in India is in intermediate range.

Every year 100,000 Indians die due to HBV infection related illnesses. Surveys for screening HBsAg have been primary, simple and the most useful mode of determining HBV infection rates.HBV transmission can occur Parenterally, Sexually or perinatally.

Certain population have been recognized who are at a greater risk of acquiring HBV infection. These include those requiring multiple transfusions, hemodialysis, renal transplant recipients, hospital staff, drug abusers, homosexuals and the immune compromised.

Since there is no intermediate host identified till now for HBV these People are high risk population and acts as carrier and transmit infection to general population. ${ }^{6,7}$ The epidemiological studies are extremely useful in identifying the HBV in the general and high risk personals to take measures for the prevention of HBV infection .

Blood transfusion service (BTS) is an integral and indispensable part of the healthcare system. The priority objective of BTS is to ensure safety, adequacy, accessibility, and efficiency of blood supply at all levels Transfusion associated hepatitis $\mathrm{B}$ viral infection (TAHBV) continues to be a major problem in India even after adoption of mandatory screening of hepatitis B surface antigen (HBsAg) by ELISA. The high incidence of TAHBV is reported in patients receiving multiple blood transfusions. A constant monitoring is required to determine the safety and prevention of infection. ${ }^{8}$
A teaching Hospital patient based study is helpful in assessing true nature of problem in the community. Prevalence studies help in assessing the magnitude of $\mathrm{HBV}$ infection and aid in devising preventive measures. This study was undertaken to estimate the burden of HBV infection among blood donors \& hospital camp attendees in a tertiary care hospital.

\section{Materials and Methods}

\section{Study Setting}

Owaisi Hospitals is a tertiary care super speciality hospital with bed strength of more than 1000.The hospital has been recognized as a referral centre by CGHS Central government health scheme and most other private Organizations. Hence there is a good opportunity to screen for $\mathrm{HBsAg}$ in blood donors attending the hospital.

\section{Study Population}

The study population included all the consented blood donors donating blood in the blood bank of the hospital and hospital camp attendees. The study was approved by Institutional ethics committee.

Study period: 6 months from February 2015 to July2015.

\section{Method of Collection}

A routine general examination was done for all the individuals coming for the blood donation and hospital camp attendees. Blood pressure, height \& weight were measured. Haemoglobin estimation was done.

Persons belonging to certain risk groups like patients from thalassemia, dialysis patients, pregnant women were excluded from the study. 
Informed written consent was taken from them study participants after explaining about the purpose and objective of the study. A pre designed questionnaire was used to collect information related to socio demographic profile. They were also asked about previous surgical procedures, history of past or current use of intra venous drugs, etc. The test results were kept confidential.

Standard procedures were used during the blood collection. A total of 2,500 donor blood samples \& 500 hospital camp attendees were screened by CDSCO approved ELISA kit Hepalisa (J Mitra\& Co Pvt. Ltd. India) and HepanostikaHBsAg Ultra.

\section{Data Analysis}

Data entry was done using Microsoft Excel 2007 version and analysed using Epi info version 7 . Frequency tables and graphs were used to represent data. Data is summarized in percentages. Univariate analysis was used with Chi-square test and significance level at $5 \%$ is used to determine the association of various independent factors.

\section{Results and Discussion}

A total of 2,500 blood donor samples and 500 hospital camp attendees were screened during the study period. Majority were males (86\%) and belonged to 26-30 years age group (43.5\%).

\section{Prevalence of $\mathrm{HBsAg}$}

Out of the total 2,500 samples studied, 30 of them were found to be positive for HBsAg. The prevalence rate was $1.2 \%$. Out of the total 500 hospital camp attendees, 22 of them were found to be positive for HBsAg. The prevalence rate was $4.4 \%$. (Table 1 )
The overall prevalence of seropositivity was more among hospital camp attendees when compared to blood donors which was found to be statistically significant (chi square 25.05, DF=1, $\mathrm{p}<0.0001$ ).

Demographic characteristics of the HBsAg positive blood donors found that majority $(93.3 \%)$ were males and half the proportion (53\%) belonged to 31-35 years age group. Occupation wise revealed that majority (50\%) were from business. In comparison with voluntary donors, seropositivity was more among replacement donors (blood donated to replace blood utilized which includes relatives or friends of patients).

Immunization status among the two groups found that blood donor group were better immunized than hospital camp attendee which was found to be statistically significant. $(\mathrm{p}<0.05)$.

The discovery of HBsAg was a major breakthrough in decreasing the incidence of post transfusion hepatitis. According to India's Drugs and Cosmetics Act (1945), each blood unit has to be tested for hepatitis $B$ virus infection. Present study which has been designed with an objective to assess the prevalence of HBsAg in blood donors and hospital camp attendees found that prevalence was $1.2 \%$ and $4.4 \%$ respectively showing a higher prevalence in hospital camp attendees.

A hospital based study by Yashovardhan et al in Andhra Pradesh observed that the overall prevalence of Hepatitis B seropositivity was $2.2 \%$. No statistical difference was observed between male \& females and seropositivity was more among voluntary blood donors compared to replacement donors in contrast to the findings of the present study. ${ }^{9}$ 
Table.1 Prevalence of HBsAg among Blood Donors\& Hospital Camp Attendees

\begin{tabular}{|l|l|l|}
\hline \multirow{3}{*}{ Result } & \multicolumn{2}{|l|}{ HBsAg } \\
\cline { 2 - 3 } & Blood donors & Hospital camp attendees \\
\cline { 2 - 3 } & No $(\%)$ & No $(\%)$ \\
\hline Positive & $30(1.2 \%)$ & $22(4.4 \%)$ \\
\hline Negative & $2470(98.8 \%)$ & $478(95.6 \%)$ \\
\hline Total & $2500(100 \%)$ & $500(100 \%)$ \\
\hline
\end{tabular}

Table.2 Characteristics of HBsAg Positive Cases among Blood Donors $(\mathrm{n}=30)$ \& Hospital Camp attendees $(\mathrm{n}=22)$

\begin{tabular}{|l|l|l|}
\hline & Blood Donors & Hospital camp attendees \\
\cline { 2 - 3 } Characteristics & No (\%) & No (\%) \\
\hline Sex & & \\
Male & $28(93.3 \%)$ & $19(86.4 \%)$ \\
Female & $02(6.7 \%)$ & $04(1.4 \%)$ \\
\hline Age & & \\
$21-25$ & $01(3.3 \%)$ & $02(9.1 \%)$ \\
$26-30$ & $02(6.7 \%)$ & $05(22.7 \%)$ \\
$31-35$ & $16(53.3 \%)$ & $10(45.5 \%)$ \\
$36-40$ & $06(20 \%)$ & $04(18.2 \%)$ \\
$41-45$ & $05(16.7 \%)$ & $01(4.5 \%)$ \\
\hline Occupation & & \\
Labourers & $07(23.3 \%)$ & $03(13.7 \%)$ \\
Business & $15(50 \%)$ & $06(27.3 \%)$ \\
Other & $08(26.7 \%)$ & $13(59 \%)$ \\
\hline
\end{tabular}

Table.3 Immunization Status against HBsAg among Study Population

\begin{tabular}{|l|l|l|l|}
\hline \multirow{2}{*}{ Group } & \multicolumn{2}{|l|}{ Immunization status } & \multirow{2}{*}{ Total } \\
\cline { 2 - 3 } & Immunized & Not Immunized & 2500 \\
\hline Blood donor group & 312 & 2188 & 500 \\
\hline Hospital camp attendees & 39 & 461 & \\
\hline
\end{tabular}

Chi square $=8.8, \mathrm{DF}=1, \mathrm{p}$ value $=0.002$

A similar kind of study by DeeptiSachan et al in Chennai found that prevalence of HBsAg positivity was similar in both blood bank donors and hospital camp attendees. ${ }^{10}$ This finding is in contrast to the present study in which prevalence of seropositivity was more among hospital camp attendees when compared to blood donors which was found to be statistically significant $(\mathrm{p}<0.0001)$.

Seropositivity was found to be $3.5 \%$ among 200 blood donors in a study by Lavanya et al in Tamil Nadu. The prevalence of HBV markers were found to be instigated with factors like alcoholism, smoking, tattooing, ear piercing, visiting barber's shop, and family history of jaundice, and were statistically significant. ${ }^{11}$

Another study by Karandeep Singh et al on trends of seroprevalence of Hepatitis B virus infection among blood donors of coastal Karnataka revealed a very low prevalence $(0.62 \%)$ with a decreasing trend over three years. Replacement donors and male blood donors showed higher seropositivity 
compared to voluntary donors and female donors. $^{12}$

In conclusion, a higher rate of prevalence of HBsAg positivity was observed among hospital camp attendees compared to blood donors. Among blood donors, it was found that Sero positivity was more among replacement donors. Immunization status among the two groups found that blood donor group were better immunized than hospital camp attendee which was found to be statistically significant. There is a need for $100 \%$ immunization for all the people especially the hospital staff at the time of recruitment itself. This can minimise the risk of acquiring the infection and decrease the prevalence.

\section{References}

1. Kar P, Budhiraja S, Narang A et al. Etiology of sporadic acute and fulminant Non-A Non-B viral hepatitis in north India.Indian journal of Gastroentrology 1997;16:43-45.

2. Lee WM.Hepatitis B virus infection. NEngl J Med1997; 337:733-45.

3. Lok AS, Health cote E J, Hoofnagle JH. Management of hepatitis B; 2000Summary of a workshop. Gastroentrology 2001; 120:1828-53.

4. Lavanchy D. Public health measures in the control of viral hepatitis. A WHO perspective for next millennium. J GastroenterolHepatol 2002; 17:452-59.

5. WHO core programme clusters. Family and Community health. Hepatitis B. Community Office for
India.(http://www.whoindia.org/en/sectio n6lsection8.htm)

6. Poustchi H, Mohamadnejad M, Malekzadeh R. Hepatitis B virus infection in Iran. Iran J Clinic Infect Dis2007;2(1):37-51

7. Zali MR, Mohaghegh SH. The changing epidemiology of hepatitis B in Iran. J Gastroenterology Hepat2009; 3(1).

8. Islam MB. Blood transfusion services in Bangladesh Asian J. Transf. Sci.2009; 3:108-110.

9. Yashovardhan A, SreedharBabu KV, Jothi Bai DS. Trend of Hepatitis B Surface Antigen (HBsAg) among blood donors at the blood bank of a tertiary care referral teaching hospital in southern India. Int J Res Med Sci 2015; 3:1686-90.

10. DeeptiSachan, Joy Varghese, JensinghJoseph,Vijaya Srinivasan, VenkataramanJayanthi, andMohamedRela. HBsAg Positive Patient Characteristics in Hospital and Blood Donation Camps. ISRN Hematology Volume 2013 pg no 1-3.

11. V. Lavanya, T. Viswanathan, S. Arul Sheeba Malar, A. Malarvizhi and K. Moorthy.Prevalence of hepatitis B virus infection among blood donors with antibodies to hepatitis B core antigen. International Journal of Medicine and Medical Sciences 2012; 4(6):128 - 137.

12. Karandeep Singh, Sudha Bhat, Shamee Shastry. Trend in seroprevalence of Hepatitis B virus infection among blood donors of coastal Karnataka, India. J Infect Dev Ctries2009; 3(5):376-379.

\section{How to cite this article:}

Md Wajid and Md Khaleel. 2016. Prevalence of Hepatitis B surface antigen among blood donors and hospital camp attendees at a tertiary care hospital, Hyderabad, India. Int.J.Curr.Microbiol.App.Sci. 5(4): 864-868. doi: http://dx.doi.org/10.20546/ijcmas.2016.504.098 